\title{
REGULATORY REQUIREMENTS IN THE GMP PRODUCTION OF AN EPITHELIAL CELL GRAFT FOR OCULAR SURFACE RECONSTRUCTION
}

\author{
Radhika Sheth-Shah $^{1,2^{*}}$, Amanda J Vernon ${ }^{1,2}$, Shankar Seetharaman ${ }^{3}$, Michael H Neale ${ }^{1,2}$ and Julie T Daniels ${ }^{1,2}$ \\ ${ }^{1}$ Cells for Sight, Transplantation and Research Programme, University College London Institute of Ophthalmology, London, UK \\ ${ }^{2}$ Ocular Biology and Therapeutics Division, University College London Institute of Ophthalmology, London, UK \\ ${ }^{3}$ Pharmanswers Ltd., Cambridge, UK \\ *Author for correspondence: radhika.shah@ucl.ac.uk
}

\begin{abstract}
:
In the past decade, stem cell therapy has been increasingly employed for the treatment of various diseases. Subsequently, there has been a great interest in the manufacture of stem cells under Good Manufacturing Practice (GMP), which is required by law for their use in humans.

The Cells for Sight (CFS) Stem Cell Therapy Research Unit, based at UCL Institute of Ophthalmology, delivers somatic cell based and tissue-engineered therapies to patients suffering from blinding eye diseases at Moorfields Eye Hospital. The following manuscript is based on our experience in the conception, design, construction, validation and manufacturing within a GMP manufacturing facility based in the United Kingdom. As such the regulations can be extrapolated to the 28 member stated within the EU. However, the principles may have a broad relevance outside the EU.
\end{abstract}

Keywords: Good manufacturing practice, cell therapy, quality control and cleanroom

\section{AN INTRODUCTION TO REGULATIONS:}

Regenerative medicine (RM) has been defined as the 'process of replacing, engineering or regenerating human cells, tissues or organs to restore or establish normal function'[1]. RM promises to revolutionize patient care in the $21^{\text {st }}$ century through an amelioration of cell and tissue replacement therapies. Stem cell research plays a dominant role in RM through translational research aimed at repair and regeneration of diseased or ageing tissues and organs. A medicinal product, used for tissue regeneration within the European Union, must comply to the European regulations on the production of human[2] and veterinary[3] medicines and pharmaceuticals. Within Europe, the manufacture of medicines is regulated by European Medicines Agency, who decrees that each EU member state is required to elect a competent authority to oversee licensing of manufacturing facilities ratifying their compliance to Good Manufacturing Practices (GMP). GMP is a quality system for ensuring that medicinal products are manufactured consistently and to defined standards thus ensuring medicines are as safe as possible. GMP has historically been employed in the production of small molecules, proteins, vaccines and monoclonal antibodies [4]. However, in 2004, the European Commission ratified the Tissues and Cells Directive (EUTCD; [5]) which re-classified gene therapy, somatic cell therapies (which includes stem cell therapy products) and tissue engineered products as ATMPs (Advanced Therapy Medicinal Products) and imposed their manufacture (i.e. expansion or modification) to apply to the same GMP principals. Two Directives lay down the principals and guidelines of GMP for medicinal products, Directive 2003/94/EC which is concerned with medicinal products of human use [6] and 91/412/EEC intended for veterinary use medicines[7].

One potential confusion within the field of cell therapy manufacturing is what regulations applies to human tissue and/or cells isolated from tissue used for direct transplantation or as part of a medicinal product. As previously mentioned, the EUTCD was issued in 2004 and one of its aims was to clarify these definitions. Where whole tissues intended for human application, are donated from the living or deceased, the consent, procurement, processing, testing, storage and disposal of that whole tissue is governed by EUTCD with UK enforcement provided by the Human Tissue Authority (HTA). Where individual cells, isolated from whole nonembryonic human tissues, are expanded or modified to make a cell based medicinal product then their manufacturing and quality control testing, would fall under the ATMP Directive 2009/120/EC [8] and the regulating authority would be the Medicines and Healthcare products Regulatory Agency (MHRA). The MHRA are the competent authority within the UK, whose remit covers the regulation of medicines, devices, blood and 
Advanced Therapy Medicinal Products (ATMPs) for human application. In the UK, manufacture of veterinary products is regulated by the Veterinary Medicines Directive (VMD).

In addition to ensuring medicines are manufactured to GMP, the MHRA also issues authorisations allowing the distribution of safe and tested medicines on the market (termed a marketing authorisation; MA), or authorises their use within a clinical trial, the medicine in this case is termed an Investigational Medicinal Products (IMP). Facilities manufacturing medicines intended for release to the market must be granted a manufacturing license (MIA) by their competent authority, whilst facilities manufacturing IMPs must also obtain a separate MIA IMP license.

In the UK, in addition to the above-mentioned manufacturing licenses (MIA or MIA IMP), ATMPs may be manufactured under a UK 'Specials' or a hospital exemption license for an unmet clinical need i.e. where no alternative licensed product is available. Cells for Sight have experience of manufacturing ATMP 'Specials'. Here, a bona fide, unsolicited request from the patient's surgeon, in the form of a prescription, is required to commence the manufacturing process. The legal responsibility for use of the ATMP in this case rests with the surgeon. The main difference between the two types of licence are; that the holder of a 'Specials' license can manufacture an ATMP for any EU member, whereas a hospital exemption holder may only manufacture ATMPs for their own hospital's patients. ATMPs manufactured as 'Specials' must meet the expectation of 2003/94/EC [6] and Guidance Note 14 [9] and may be released by the Quality Controller (QC) of the manufacturing facility as opposed to the Qualified Person (QP).

At CFS, we manufacture ATMP MIA-IMPs generated from expended stem cells to produce novel cell-based and tissue-engineered products. Medicinal products must be certified by a QP prior to release. The QP plays an integral role in medicine manufacture and must take final responsibility for the release of that product for human or animal use. The QP must ensure the product is safe by ratifying it has been manufactured to GMP and in accordance with the marketing authorisation (for products released to the market), or in accordance with an IMP Dossier (IMPD) and Product Specification File (for IMPs).

Irrespective of the type of medicine being produced, and the type of license a manufacturing facility holds, the principles of GMP must always be applied in order to ensure medicines are consistently produced, of the highest quality, safe and effective for the end user. Under the EU regulations, ATMPs, which are classified as sterile medicinal products, must be manufactured within a cleanroom to ensure no contamination is transferred to the recipient. There are many requirements of a cleanroom in order for it to be 'fit for purpose' and the following section describes these in detail.

\section{CLEANROOMS:}

A cleanroom is a laboratory in which the level of particulate and microbial contamination is controlled.

In Europe, Eudralex Volume 4 Annex 1 recognizes four clean room grades based on the maximum permitted number of particles per $\mathrm{m}^{3}$ and microbiological load limits [10]. These are listed in Table 1:

Practically, the grades are distinguished as (Table 2):

The environmental conditions within the cleanroom are controlled by a sophisticated Heating Ventilation and Air Conditioning (HVAC) unit, which controls the temperature, humidity, differential pressures between rooms and particulate content of the air. Clean air within the manufacturing facility is generated by a series of High Efficiency Particulate Air (HEPA) filters that deliver pressurised air, via a dedicated air-handling unit, to each room. Air is filtered through a series of pre-filters, panel filters and a terminal HEPA which is classified by its efficiency to remove its most penetrating particle size. At CFS the terminal HEPA is classified as H14 which means it removes $99.995 \%$ of particulates $0.1-0.3 \mu \mathrm{m}$ [11]. A pressure differential of 10-15 Pa between successive rooms creates a clean air cascade, which ensures that the air grade of each room is maintained. This pressure differential also ensures that particles within each room are carried outward with the outward flow of 
air. Where microbial cross contamination is not a concern, the air can be recirculated; however, due to pressure drops through natural seepage, supplementation by filtered fresh air supplied from an AHU (Air Handling Unit) is required. The air exhausted from the cleanroom is returned to the AHU (or in the case of single pass systems vented to the outside). The exhaust may also be HEPA vented depending on the type of material (e.g. viruses or GMOs) being manufactured.

Generally, the airflow within the cleanroom is either turbulent or laminar. Laminar is uni-directional and involves a continual downward constant stream of air. Most cleanrooms are built with this design, however, some are turbulent flow which is not uni-directional and this method is used to move particles around the facility until it ultimately drives them to the floor and towards the extracts. Turbulent flow cleanrooms are allowed for cell therapies when manufacture is performed under a laminar flow cabinet or bench.

Where protection of the product is required, such as the cell therapy products manufactured at CFS there must be a positive pressure air gradient, whereby the air moves outwards from the Grade A/B laboratory in order to minimize environmental contamination of the product. For gene therapy products, or those containing high potency drugs, the primary processing Grade A/B laboratory is maintained under negative pressure surrounded by positive pressure gradients; for containment purposes. This is usually done through an air sink outside the processing room.

\section{CONSTRUCTION OF THE GMP FACILITY}

From a regulatory point of view, premises must be designed, constructed, located and maintained to suit the operations to be carried out. Materials used to line the floor and walls should be impervious to liquids and not release particles. Tiles should be avoided as gaps between tiles allow space for contamination to settle within and are also hard to clean. Internal surfaces should smooth and suitable for cleaning. The AHU should be situated outside the GMP facility so as to minimise the chance of contamination during its servicing and maintenance. Overall, the facility should be ergonomically designed to minimise the likelihood of operator error whilst allowing room for effective cleaning and maintenance so as to avoid build-up of particulates and crosscontamination that could adversely affect product quality.

Once the facility is structurally built, the internal environment must be established. For cell therapy facilities, HEPA filters are usually installed in the ceiling with an extract near the floor which ensures that the majority of the facility is always exposed to airflow. Pre-filters are generally installed in order to reduce the particle load on the HEPA filters. Pre and HEPA filters should be regularly serviced and checked to ensure that no particulate matter makes its way to the cleanroom facility. For optimum performance, pre and HEPA filters should be changed as per the manufacturer's instructions. Lifespan of these filters pre-dominantly depends on the surrounding environment for eg. filters in an urban area might get dirtier compared to filters installed in a facility based in a rural landscape.

Typically, the preferred method for entry/ exit within the cleanroom is the one-way flow of personnel, consumables and waste as it prevents room re-entry, therefore, minimising the risk of cross-contamination. Exit occurs through a separate de-gowning room. However, this setup is not always possible; therefore, backtracking through the previous rooms is allowed, albeit with extra precaution. Pass through chambers are generally built within the walls to allow for transfer of consumables, reagents and waste through the facility. Both the product and the waste can be transferred through the pass through chambers; however, they should be physically segregated.

\section{QUALIFICATION PROCESSES IN THE VALIDATION OF A CLEANROOM:}

Cleanrooms have to be validated and undergo a process of qualifications (Fig. 1). Only once it has passed all these requirements, can it be licensed for use in the manufacture of ATMPs destined for human application.

\section{EQUIPMENT:}


Only equipment necessary to manufacture the medicinal product should be installed within the facility. At the outset, equipment should be validated, either by the supplier or the manufacturing team, to ensure it is fit for purpose and does not compromise product quality. The equipment should be placed in such a way that it does not obstruct the airflow within the facility. Post installation, all equipment must be serviced and calibrated as necessary to ensure that the quality of products is not compromised. Details of equipment specification, performance criteria, servicing and calibration must be retained for future reference.

\section{PERSONNEL:}

Of importance to every GMP facility are the Personnel who physically manufacture the product as per written procedures. Therefore, it is essential that there are sufficient personnel available to carry out all the tasks required for manufacture of the product. All personnel should be aware of and trained to their specific tasks. Training records for each individual should be generated and filed. Competence for all GMP related tasks including cleaning, gowning, aseptic technique etc should be documented on the training record.

Each facility should have an organisational chart in which the responsibilities and the relationships between each individual are explicitly defined. Most GMP facilities are divided into two departments: Production and Quality Control/ Assurance; each as independent from the other as possible [12]. The production team is trained in and is responsible for all aspects of manufacturing the product. The QC department does not get involved in processing operations but does monitor and review the Quality Management System (QMS), approve starting materials, ensure testing and validations are carried out and reviews product related documentation/ investigations. The QC department may have a Quality Assurance (QA) unit which may perform all, or specific, aspects of the above dependent upon the companies' organisation. In smaller facilities, both the QC and QA responsibilities maybe managed by the same individual.

\section{FACILITY MONITORING:}

The facility and equipment should be regularly monitored to ensure that there is minimal risk to the product quality. Ideally, an automated system should be installed to ensure continuous monitoring of all parameters. This should be supplemented by manual monitoring to ensure that any fault within the automated system is promptly detected without any effect on the product quality. Various parameters need to be monitored to ensure that the facility and the equipment housed within are functioning within their set specifications, these include but are not limited to: room temperatures and humidity, room pressures, pressure differentials, particle levels within the facility and safety cabinets, temperature, $\mathrm{CO} 2$ and humidity of the incubators, temperature of the fridge/ freezers etc. Each parameter should be continuously monitored and should additionally be manually checked during working hours.

Monitoring particle counts is extremely important as particles may serve as a vector for attachment of various micro-organisms which pose a risk of contamination to the product. Particles, by themselves, can also contaminate the product. Related to the particle counts are the pressure differentials as a positive pressure cascade ensures that particles are removed from the GMP facility in an outward fashion. Equipment parameters are important as they maintain the integrity of the product and/ or reagents.

\section{MAINTENANCE OF THE CLEANROOM}

Following the qualification process, cleanrooms must be maintained to ensure they are 'fit for purpose'. This includes ensuring personnel are trained to cleanroom procedures and ensuring environmental monitoring and cleaning is routinely performed.

\section{Cleaning}


A documented cleaning routine is used to minimise the risk of particulate or microbiological contamination of products resulting from airborne and surface contaminants. The effectiveness of the cleaning routine is determined by regular environmental monitoring according to a documented series of validated procedures. Cleaning includes all surfaces, equipment, consumables, doors, walls and floor. It should be performed by trained personnel and be thoroughly documented.

At CFS, cleaning of the manufacturing facility is performed on an in-use basis, a weekly basis, a monthly basis and a quarterly basis. 'Critical areas', i.e. those directly involved in product processing are cleaned on an in-use basis. 'General areas', i.e. those not directly involved in production but which present a low risk of contamination to critical areas via transfer, are cleaned with sterile 70\% IPA (Isopropyl Alcohol) according to a weekly, monthly and quarterly schedule, with alternating Biocide A (bactericidal and fungicidal), B or C (bactericidal, fungicidal and sporicidal virucidal) cleans on a monthly basis. If environmental monitoring results indicate that additional measures are required then extra ad hoc cleaning with Biocide $\mathrm{A}, \mathrm{B}$ or $\mathrm{C}$ is performed. Biocides must be alternated to avoid development of resistance within organisms. Deep cleaning e.g. on an annual basis or following a persistent contamination resistant to routine cleaning may be performed with vapour hydrogen peroxide. To minimise particles within the facility, cleaning reagents are sprayed onto sterile low particle shedding wipes and then the reagent is wiped onto the surface being cleaned using unidirectional overlapping strokes to ensure that the whole area is cleaned. Ceiling, walls and floors are cleaned with a mop head attached to a mop. Other facilities may use pre-impregnated wipes for surfaces and equipment, or other validated cleaning systems. IPA is allowed to evaporate over time while biocides are left in contact with surfaces for 5 minutes. Some Biocides leaves residues, and for these a second clean with IPA is performed. Biocides may be corrosive by nature, therefore, care should be taken to ensure that the biocide does not dry out and leave behind any residue.

Large facilities tend to use pre-impregnated wipes for surfaces and equipment. This is advisable for large facilities as it is more cost effective whereas in smaller facilities, the impregnated wipes may dry out if left opened for days, leading to waste of reagents and money.

\section{Environmental Monitoring (EM)}

To establish whether the cleaning protocols meet the required standards with respect to control of particulate and microbiological contamination within the production areas, routine environmental monitoring is performed according to documented procedures. This is performed both 'in-use' to determine the level of contamination present during production, and 'at-rest' on a weekly basis to determine the effectiveness of cleaning routines. EM utilises a number of agar plates to monitor bacterial and fungal contamination, at CFS, Tryptone soya agar (TSA) and Sabaroud dextrose agar (SDA) plates are utilised respectively for this purpose. The plates themselves are of two different types: Settle and contact (Fig.2). Contact plates themselves are used either to perform contact monitoring or active air sampling.

Settle plate monitoring is a direct method for evaluating the approximate number of microorganisms depositing on the agar surface in a set amount of time; from air within the cleanroom.

Contact plate monitoring is a method of surface sampling performed in order to determine the number of microorganisms/ contamination on any surface within the cleanroom or upon the operator's apparel. Contact plates normally have a raised media surface to allow for direct application to the test surface.

The air circulating within the cleanroom is also monitored by an active air sampler which captures air through a high pressure inlet. The captured particles collide against the agar surface and settle on it.

At CFS, EM plates are incubated at specified temperatures $\left(35^{\circ} \mathrm{C}\right.$ for bacteria and $22^{\circ} \mathrm{C}$ for fungi) for $3-5$ days and checked for colony forming units ( $\mathrm{cfu}$ ) to determine the number of microbe bearing particles deposited onto the agar. Positive and negative controls are also prepared to ensure the accuracy of results. Results from 'at rest' and 'in-use' environmental monitoring are recorded in a spreadsheet in order to determine any contamination trends. This information is used to determine if there is a need for corrective and preventive action, according to whether action levels or alert levels have been reached. Action levels are indicative of unacceptable levels of microbiological contamination and require corrective and preventive action to be taken in accordance with 
written procedures. Action levels are those defined in 'Rules and Guidance for Pharmaceutical Manufacturers and Distributors 2007' [13]. Alert levels are set at 50\% less than action levels, for which no action is necessary. Rather, the problem area is noted, its cleaning detail is improved, and personnel performance is evaluated to determine whether re-training is necessary.

\section{TRAINING}

\section{Personnel}

Personnel are also a significant source of contamination. Therefore, traffic of personnel, visitors and engineers is strictly controlled to minimise the risk of particulate and microbiological contaminants entering the manufacturing facility. Cleanroom entry, gowning and hygiene routines are implemented according to validated procedures, in which all users of the facility are trained. Visitors and engineers are given training appropriate to their task prior to entry within the cleanroom.

All users of the facility are also required to adhere to some basic behavioural and personal disciplines in order to minimise particle shedding and potential contamination, these include:

- Maintenance of a good level of personal hygiene, including showering regularly, keeping dandruff at bay and moisturizing dry skin to reduce skin shedding

- No cosmetics, talcum powders, hair sprays, nail polish or similar materials

- No jewellery or watches within the cleanroom

- Controlled movements within the cleanroom, with no rushing and to refrain from any sudden/ jerky movements

In terms of particle shedding, movement is perhaps the greatest factor as the number of particles shed is proportional to the level of activity performed, with 100,000 particles $\geq 0.5 \mu \mathrm{m} / \mathrm{min}$ shed while sitting, rising to 1 million when moving and 5 million when walking [14]. Therefore, slow, controlled movements within the cleanroom facility are essential to minimise aberrant particles.

\section{Gowning:}

Outdoor clothing should not be brought into the facility beyond the Grade C/D changing room. For wearing within the Grade A/B areas, sterilised protective garments should be provided (Fig. 3). Personnel must be trained to change clothing in accordance with a gowning procedure in order to minimise particle shedding and potential contamination within the facility.

At CFS, all garments are sterilised by gamma irradiation by a specialist laundering company and are delivered triple wrapped. Each garment is single use and is sent back to the laundering company to be cleaned and resterilised a finite number of times after use.

In order for an operator to perform any processing in the facility they need to have passed a gowning training test, this involves gowning in according to cleanroom techniques to enter the grade B laboratories. Gowning is followed by a series of microbial monitoring contact plates to assess levels of contamination on areas of gowning which may come into contact during the gowning process - gloves, forearm, forehead, chest, and stomach. If the plates have passed i.e. no CFU on the contact plates, the operator is allowed to process in the facility.

\section{Aseptic training}

All staff members who work in the manufacturing facility, including those involved in product manufacture are trained in aseptic technique on recruitment and at regular intervals thereafter. 
At CFS, all operators are required to pass a training module to demonstrate aseptic technique within the biological safety cabinets (Grade A environment) - this involves a three day process of manipulating cell culture plates or flasks and liquid handling, transferring sterile tryptone soya and sabouraud dextrose broths (TSB and SDB, respectively) between wells on three separate days and the inoculation of further TSB and SDB for subsequent incubation for $3-5$ days and analysis for turbidity. Additionally in-use monitoring is also performed assessing the working environment and the operators' gloves for contamination. In order to pass this training module, all broths should be free from turbidity and monitoring plates should be below allowable limits (Table 3). This training module is repeated every 6 months and results recorded in the operators training record. This procedure is specific to CFS, other facilities will need to simulate training appropriate to the processes to be performed within their respective facilities.

\section{QUALITY MANAGEMENT SYSTEMS (QMS)}

A Quality Management System (QMS) has the purpose of assuring product specification and quality through the control of inter-connected elements and to mitigate potential risks to the product.

QMS is based upon International Organisation for Standardisation (ISO) 9001:2008, a set of standards originally developed in 1987 to maintain quality assurance in product manufacturing and service industries. The pharmaceutical industry has further developed these standards through the International Conference on Harmonisation (ICH), to produce a harmonised comprehensive model for an effective pharmaceutical quality system designed for the entire product lifecycle.

Within the pharmaceutical industry, a QMS is the requirement of Directive 2003/94/EC (manufacture of medicinal products for human use); [6] and Directive 91/412/EEC (manufacture of medicinal products for veterinary use); [7], and ensures the principals and guidelines of these Directives are implemented, including compliance to GMP.

All further reference to the QMS within this publication will be based on the CFS QMS, unless otherwise specified. The QMS defines how the activities performed during ATMP manufacture ensure compliance to local regulatory requirements of the MHRA and HTA. The CFS QMS is organised into the following categories: Validations; Donor Recruitment and Patient Treatment; Production and Quality Control; Quality Assurance, where each category comprises of a set of Standard Operating Procedures (SOP's) that provide a top-level description of how the QMS is implemented (Fig. 4).

Additional to the SOPs, various documented procedures (including Quality Assurance Forms, Training Forms, Corrective and Preventive Action Procedures, and Production Protocols) are used to execute the QMS for the manufacture of ATMPs.

The following describes the requirements and the implementation of the various sections of the QMS and how it interacts with GMP compliance and human tissue regulations.

Validations are required to be performed for a process, reagent, a piece of equipment or for the manufacturing facility itself in order to ensure the parameter in question is 'fit for purpose' and will not affect product quality once initiated or implemented. Following quality approval, successful validations allow the parameter in question to then be built into the QMS through updates to existing GMP documentation or implementation of new GMP procedures.

A section of the QMS is dedicated to Donor Recruitment and Patient Treatment to ensure the CFS facility, when manufacturing ATMP's using autologous or allogeneic donor tissue and cells, complies to the legal framework (Human Tissue Act 2004 [15], the Human Tissue Regulations 2007 [16] and EU Tissues and Cells Directives [5]) that dictates procedures surrounding consent for tissue donation, procurement, testing, storage, disposal and use of body parts, organs and tissue .

The QMS also details policies around production and quality control activities. The Production SOP documents the required production procedures to be performed to maintain GMP standards. Such procedures include; 
facility clean, facility and environmental monitoring, equipment planned preventative maintenance (PPM) and QC sterility assessments for aliquoted reagents.

Quality Assurance (QA) is directly concerned with assuring the quality of a product through controls that are described in a set of SOPs. Quality Assurance is also concerned with monitoring procedures and processes, providing a feedback loop to aid improvements in order to prevent error in the future. Although each of the aspects detailed in Fig. 3 is essential to the smooth running of a cleanroom, only the critical sections will be discussed here.

The investigation of deviations forms a major part of any organisation's strategy for risk management and quality improvement. Where a deviation or non-conformance occurs within a prescribed process, the incident needs to be recorded and corrected. The term 'deviation' is used to include process deviations, planned changes, out of specification events and any other non-compliance with GMP. This overall system includes; recording of the deviation, assessment of the deviation in terms of risk to the facility, product or patient, root cause analysis, planning and implementation of Corrective and Preventative Actions (CAPA) and routine trending of deviations so that higher levels of preventative action can be taken.

A complaint is defined as any defect in a product or service reported by either an external or internal customer. The Complaints and Recalls SOP defines the process employed which includes risk and impact assessment, root cause analysis and implementation of CAPA to prevent a similar issue occurring in the future.

Change Control is the process that defines the way in which changes to all aspects of the QMS are managed. In practice the following require change control: starting materials, documents, equipment, processes, any change that has a regulatory impact e.g. key staff changes, new product types, facility changes and any other change that may affect the quality of the product. Changes can occur in response to many parameters for example; deviation, complaint, audit, introduction of a new project, or contract change. Change requests are trended and reviewed periodically to help identify potential improvements.

A Service level Agreement (SLA) must be in place when an organisation provides a service to, or is provided with a service from, a third party. The SLA will establish the expectations and requirements that should be met by both parties. Should we define the different one?

Before a medicinal product can be used to treat a patient, it must be formally released for use by CFS and therefore only officially released products can be used to treat a patient. The Batch Release SOP documents the procedures by which a product is released for patient use including; review of batch records, test results, deviation reports and monitoring results culminating in a formal sign-off by the QP for IMP products or the QA Manager for 'specials' products.

The Materials Management SOP documents the controls in place to mitigate potential risks to the product due to materials used within GMP manufacture. The controls described within this SOP include the following; goods/supplier selection and risk assessment, TSE compliance assessment, goods ordering and receipt, traceability and stock rotation/storage/reconciliation.

As previously described, all of the above factors have to be stringently controlled in order to ensure medicines (including cell therapy products) are manufactured safe, effective and of the highest quality. Below is an example of how a CFS product is manufactured in adherence with EU regulations and the CFS procedures described above.

\section{LIMBAL EPITHELIAL STEM CELLS AND LIMBAL STEM CELL DEFICIENCY:}

CFS have worked with academic, industry and NHS partners in the manufacture of cell therapy products. One particular cell based therapy manufactured by CFS is used to treat patients' suffering from blinding eye diseases such as limbal stem cell deficiency (LSCD) which results in corneal degeneration. 
The cornea is the clear, front surface of the eye that transmits light to the retina to enable vision. Corneal epithelium is separated from the conjunctival epithelium by a narrow band of tissue known as the limbal epithelium. Limbal epithelial stem cells (LESC) reside within the limbal epithelium and play a role in the maintenance and renewal of the corneal epithelium [17]. Dysfunction or damage of the limbal epithelium can lead to either unilateral or bilateral LSCD, which is characterized by vascularization, conjunctival ingrowth and inflammation resulting in visual impairment [18]. LSCD can be treated by replacement of the lost or damaged LESC by autologous or allogeneic transplantation of limbal tissue. CFS devised a technique utilizing a fully reproducible fibrin gel substrate for the culture of autologous or allogeneic limbal and autologous oral mucosal epithelial cells [19].

Described below is a brief explanation of the production of a GMP compliant cell therapy graft within CFS.

\section{GRAFT PREPARATION:}

As previously described, the implementation of any process within the cleanroom requires validation. At CFS, the graft production process was conceptualized and optimised in the research lab before finally, performing a process validation within the cleanroom facility. The process of graft production within the cleanroom is described below. The flow of the production process can be observed in Figure 5.

\section{Prescription and Patient selection criteria}

The process is initiated when the CFS team receives a prescription from the clinician requesting a specific 'Specials' product. The clinician obtains patient consent, and with them completes donor selection and testing forms which include lifestyle and medical questionnaires and also includes testing at minimum for HIV 1 and 2 , hepatitis B and C and Syphilis [20]. There is generally no difference in virology testing between autologous or allogeneic donors so as to ensure that tissue coming into the cleanroom facility is identically screened. An exception would occur where patients have travelled to 'at-risk' countries or where the behaviour and lifestyle questionnaire indicates further testing maybe required as per the guidelines. The results of a blood sample for serological screening may be obtained in sufficient time, prior to tissue donation, to allow assessment of infection risk to the GMP facility. In the UK, a second blood sample for serological screening should be obtained on the day of tissue donation (or within 7 days post donation if the former is not possible) in accordance with HTA Direction 003/2010 [21].

\section{Cell culture media}

As there is no universally accepted list of suitable materials and reagents for the manufacture of ATMPs, each facility must perform a risk assessment (RA) to ensure that the components used pose the least risk to the patient. RA addresses various criteria including tests for viruses, bacteria, fungi, mycoplasma and endotoxins to justify the use of the product. Currently available cell culture reagents are mostly approved for research use only. Therefore, the RA must demonstrate why the reagents in cell culture media are justified for use, as per the regulations. Manufacturers should provide all quality certificate available (i.e. Certificates of Analysis, Origin and TSE/ BSE compliance) upon which, these assessments will be made.

\section{Cell culture process}

Cell culture media for clinical grafts were prepared on a weekly basis to avoid any contamination during the cell culture process. Therefore, all the media components need to be aliquoted and pass QC prior to the start of cell culture. Once all the media components have passed their sterility testing, a limbal biopsy (autologous or allogeneic) or an autologous oral mucosal biopsy, as determined by the consulting clinician, may be accepted to commence cell therapy manufacture.

In the past, CFS manufactured epithelial cell grafts for patients using human amniotic membrane as described in [22]. However, due to batch-to-batch variability in amnion [23], CFS developed an alternative technique utilizing a CE marked fibrin substrate for culture of epithelial cells [19]. This technique is currently being used by CFS for manufacture of epithelial cell grafts for ocular surface reconstruction. 
The tissue culture process is set up as previously described in. The cells are fed with freshly prepared media every 2 days. Cell morphology, percentage of confluence and media turbidity are recorded at every feeding session.

\section{Critical Quality Parameters}

To release the epithelial cell graft, critical quality parameters must be met. These include but are not limited to adherent and healthy epithelial cells with cobblestone morphology, integrity of the fibrin gel, no contamination.

The graft is released for transplantation only if all of the above criteria are met. The release is authorized by the QA manager who reviews all of the batch records and associated testing results.

\section{Packaging the graft}

The cell culture media on the graft is replaced by transport media, in our case L-15, which supports the cells in environments without $\mathrm{CO} 2$ supplementation, the culture plate has an adhesive primary label attached to it and the graft is packaged in a clinical pouch sealed with GMP compliant tape. The plate is then placed into a labelled transport box. The labels and the pouch are marked with details of the patient, the cell therapy product type, expiry date and expiry time of the graft and facility contact details. Upon completion of the batch release process, the graft is delivered to the hospital for transplantation onto the patient's eye. Upon exchange of the graft to hospital personnel a 'chain of custody' form is completed to document the final stage in the process where the graft becomes the clinical teams' responsibility from this point.

\section{Post release sterility data}

The final media sterility data is only available post transplantation. In the event of a positive result, the hospital and the MHRA should be alerted, as per their guidelines. The microorganism would be identified and the species communicated to the clinical team who would treat the patient as per the clinicians' directive.

\section{Patient related data storage}

All documentation should be securely stored for the period of time defined by the directives under which the work is being performed.

\section{FUTURE PERSPECTIVES:}

Stem cell research has the potential to deliver a host of new cell therapies. The European Medicines Agency has recently granted marketing authorization to the first ever commercial stem cell therapy to treat LSCD. With increasing research progressing towards clinical trials, there is an ever increasing need for an understanding of the regulations in the production of cell therapies. Due to the vast range of possible cell therapies, it is difficult for regulators to provide guidance on specific developments within the field. Reviews like this are likely to be beneficial for future manufacturing facilities and users as they would bridge the gap between understanding and implementing the various regulations and guidelines involved in the manufacture of cell therapies. This review is also beneficial for researchers planning ATMP manufacture or clinical trials to understand the additional requirements in transferring the cell culture process from the research laboratory to the GMP facility.

\section{EXECUTIVE SUMMARY:}

\section{Regulatory Requirements within the United Kingdom}

- For manufacturing cell therapies, compliance to various legislations announced by the European Medicines Agency is required. The most important directives describing the manufacture of cell-based medicinal products for human use are Directive 2001/83/EC, Directive 2004/23/EC and 2003/94/EC.

- Where whole tissues intended for human application, are donated from the living or deceased, the consent, procurement, processing, testing, storage and disposal of that whole tissue is governed by EUTCD with UK enforcement provided by the Human Tissue Authority (HTA). 
- Where individual cells, isolated from whole non-embryonic human tissues, are expanded or modified to make a cell based medicinal product then their manufacturing and quality control testing, would fall under the ATMP Directive 2009/120/EC and the regulating authority would be the Medicines and Healthcare products Regulatory Agency (MHRA).

\section{GMP facility construction and maintenance}

- The GMP facility should be constructed in a way that it is easy to maintain. The materials used in the construction should be low particle shedding and not allow space for contamination/ dust to collect i.e. be easily accessible to allow for cleaning to occur.

- Cleaning processes should be validated and performed accordingly. The cleanroom should be periodically monitored to ensure that the cleaning protocols are effective and that the environment possesses no risk to the product quality.

- Well qualified and trained staff should be employed to reduce the occurrence of errors

\section{Quality Management System}

- All licensed manufacturing facilities should have a Quality Management System (QMS) which defines how the requirements of the specific regulatory agencies are enforced to ensure that cell therapy production is within the required legislation. A QMS will typically include policies around GMP non-compliance reporting, validations, materials management, quality assurance procedures, complaint reporting and change control to name but a few.

\section{Cell therapies for limbal stem cell deficiency (LSCD)}

- CFS, as a licensed facility, delivers cell based therapies to patients suffering from blinding eye diseases such as limbal stem cell deficiency ensuring compliance to all of the above. Limbal stem cell deficiency occurs when the cornea, the front surface of the eye, degenerates due to the dysfunction of the limbal stem cell population. CFS have developed an LSCD therapy consisting of epithelial cells in combination with a novel fibrin gel substrate. The manufacturing process includes stringent procedures and controls in order to ensure the end product is both consistent and of the highest quality.

\section{ACKNOWLEDGMENTS:}

This work was supported by the Special Trustees of Moorfields Eye Hospital and in part by National Institute of Health Research Biomedical Research Centre at Moorfields Eye Hospital NHS Foundation Trust and UCL Institute of Ophthalmology.

\section{DECLARATION OF INTEREST}

The authors have no other relevant affiliations or financial involvement with any organization or entity with a financial interest in or financial conflict with the subject matter or materials discussed in the article. No writing assistance was utilized in the production of this article. 


\section{REFERENCES}

1. Mason C, Dunnill P. A brief definition of regenerative medicine. Regen Med 3(1), 1-5 (2008).

2. DIRECTIVE 2001/83/EC OF THE EUROPEAN PARLIAMENT AND OF THE COUNCIL OF 6 NOVEMBER 2001 ON THE COMMUNITY CODE RELATING TO MEDICINAL PRODUCTS FOR HUMAN USE 67-128 (2001).

**Directive describing the practices related to the use of medicinal products in humans, within the European Union.

3. DIRECTIVE 2001/82/EC OF THE EUROPEAN PARLIAMENT AND OF THE COUNCIL of 6 November 2001 on the Community code relating to veterinary medicinal products. 1-66 (2001).

4. Sheu J, Klassen H, Bauer G. Cellular manufacturing for clinical applications. Dev Ophthalmol 53 178-188 (2014).

5. DIRECTIVE 2004/23/EC OF THE EUROPEAN PARLIAMENT AND OF THE COUNCIL of 31 March 2004 on setting standards of quality and safety for the donation, procurement, testing, processing, preservation, storage and distribution of human tissues and cells. 48-58 (2004).

*Directive describing the standards of quality and safety for the donation, procurement, testing, processing, preservation, storage and distribution of human tissues and cells, within the European Union.

6. COMMISSION DIRECTIVE 2003/94/EC of 8 October 2003 laying down the principles and guidelines of good manufacturing practice in respect of medicinal products for human use and investigational medicinal products for human use. 222-226 (2003).

**Directive describing the rules of good manufacturing practice for use of medicinal and investigational medicinal products in humans, within the European Union.

7. COMMISSION DIRECTIVE of 23 July 1991 laying down the principles and guidelines of good manufacturing practice for veterinary medicinal products 70-73 (1991).

8. COMMISSION DIRECTIVE 2009/120/EC of 14 September 2009 amending Directive 2001/83/EC of the European Parliament and of the Council on the Community code relating to medicinal products for human use as regards advanced therapy medicinal products. 3-12 (2009).

**Directive detailing the use of medicinal products described as Advanced Therapy Medicinal Products in humans, within the European Union.

9. MHRA Guidance Note 14: The supply of unlicensed medicinal products ("specials"). (2014).

10. Manufacture of Sterile Medicines. (2003).

11. Whyte W. Cleanroom Technology Fundamentals of Design, Testing and Operation. John Wiley and Sons, Ltd, 110 (2003).

12. Rules and Guidance for Pharmaceutical Manufacturers and Distributors. (8). Pharmaceutical Press, 34-35 (2014).

*Also known as the 'Orange Guide'. The book describes in detail the application of Good Manufacturing Practice for the manufacture of pharmaceutical or cell therapy products, as defined by the Medicines and Healthcare products Regulatory Agency, within the United Kingdom.

13. Rules and Guidance for Pharmaceutical Manufacturers and Distributors. (7). Pharmaceutical Press, 86-89 (2007).

14. Whyte W. Cleanroom Technology Funadamentals of Design, Testing and Operation. John Wiley and Sons Ltd., 199 (2003).

15. Human Tissue Act. (2004).

**The Human Tissue Act regulates the removal, storage, use and disposal of human bodies, organs and tissue, within the United Kingdom.

16. The Human Tissue (Quality and Safety for Human Application) Regulations 2007. 1523 (2007). 
** These Regulations describe the standards of quality and safety for human tissues and cells intended for human application. The Directives do not apply to human organs, blood or blood components.

17. Daniels JT, Dart JK, Tuft SJ, Khaw PT. Corneal stem cells in review. Wound Repair Regen 9(6), 483-494 (2001).

18. Puangsricharern V, Tseng SC. Cytologic evidence of corneal diseases with limbal stem cell deficiency. Ophthalmology 102(10), 1476-1485 (1995).

19. Sheth R, Neale MH, Shortt AJ, Massie I, Vernon AJ, Daniels JT. Culture and Characterization of Oral Mucosal Epithelial Cells on a Fibrin Gel for Ocular Surface Reconstruction. Curr Eye Res doi:10.3109/02713683.2014.978477 1-11 (2014).

20. Guide to Quality and Safety Assurance for Human Tissues and Cells for Patient Treatment. (2010).

* This guide describes the requirements for licenced facilities storing tissues and cells for human application and for licenced facilities or third parties (under an SLA) carrying out the procurement, testing, processing, distribution, import or export of tissues and cells for human application.

21. Directions given under the Human Tissue Act 2004 implementing the Human Tissue (Quality and Safety for Human Application) Regulations 2007. (2010).

22. Shortt AJ, Secker GA, Rajan MS et al. Ex vivo expansion and transplantation of limbal epithelial stem cells. Ophthalmology 115(11), 1989-1997 (2008).

23. Shortt AJ, Secker GA, Lomas RJ et al. The effect of amniotic membrane preparation method on its ability to serve as a substrate for the ex-vivo expansion of limbal epithelial cells. Biomaterials 30(6), 1056-1065 (2009).

24. Rules and Guidance for Pharmaceutical Manufacturers and Distributors (8). Pharmaceutical Press, 75-76 (2014). 


\section{FIGURES:}

\begin{tabular}{|c|}
\hline $\begin{array}{l}\text { USER REQUIREMENT SPECIFICATION } \\
\text { Provides a structure whereby evidence of compliance with user requirements can be obtained and documented }\end{array}$ \\
\hline $\begin{array}{l}\text { DESIGN QUALIFICATION } \\
\text { Provides a structure whereby evidence of compliance with the URS can be obtained and documented }\end{array}$ \\
\hline $\begin{array}{l}\text { INSTALLATION QUALIFICATION } \\
\text { Provides a structure whereby evidence of compliance with the DQ can be obtained and documented }\end{array}$ \\
\hline $\begin{array}{l}\text { OPERATIONAL QUALIFICATION } \\
\text { Provides a structure whereby evidence of compliance with all aspects of the system that affect product } \\
\text { quality operate as intended throughout all anticipated and tested ranges can be obtained and documented. }\end{array}$ \\
\hline $\begin{array}{l}\text { PERFORMANCE QUALIFICATION } \\
\text { Provides a structure whereby evidence of compliance of the facility operating within the environmental parameters } \\
\text { in the Rules and Guidance for Pharmaceutical Manufacturers and Distributors } 2007 \text { can be obtained and documented }\end{array}$ \\
\hline
\end{tabular}

Figure 1: Validation of the cleanroom. Validation of the cleanroom will include a user requirement specification (URS) which is a high level requirement and expectations for systems to be used as the basis of testing during the Performance and/or Operational Qualification (PQ/OQ) phases. The URS leads to a design qualification (DQ) which is a document that defines all hardware and software components to be installed and the configuration management plan needed to meet the URS and/or owned project requirements. This in turn would lead to an installation qualification (IQ) which is the documented verification that all aspects of the system that can affect product quality adhere to approved specifications and are correctly installed. Commissioning of the area follows the IQ operating qualification and is a well-planned and documented systematic challenge and verification using inspection, testing and documentation that a facility, system and/or piece of equipment is constructed, installed, started and functionally operates together, through normal modes and conditions in accordance with design and installation requirements and acceptance criteria. After commissioning, the operating qualification (OQ) follows which is the documented verification that all aspects of the system that can affect product quality operate as intended throughout all anticipated and tested ranges. Finally the performance qualification (PQ) is performed which is the documented verification that all aspects of the system that can affect product quality repeatedly and consistently perform as intended, meeting pre-determined acceptance criteria. 


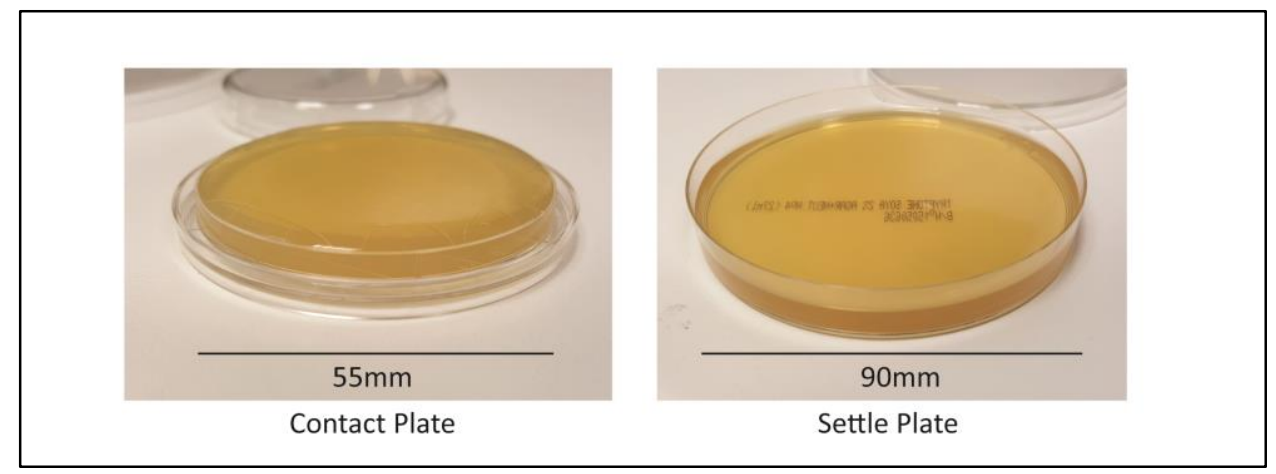

Figure 2: Examples of a contact and settle plate for environmental monitoring. At CFS, both plates are filled with either tryptone soya agar, or Sabouraud agar depending on the requirements of the monitoring. The agar also contacts various chemical neutralisers to inhibit the action of biocides to prevent false negatives. The contact plates have a raised agar surface to allow monitoring of surfaces without the side of the dish preventing contact being made (such as cabinet surfaces or glove prints form operators) whereas the settle plates are used for passive monitoring of the air within the cleanroom. Additionally the contact plates are used in conjunction with an active air sampler to actively monitor air contamination when required. 


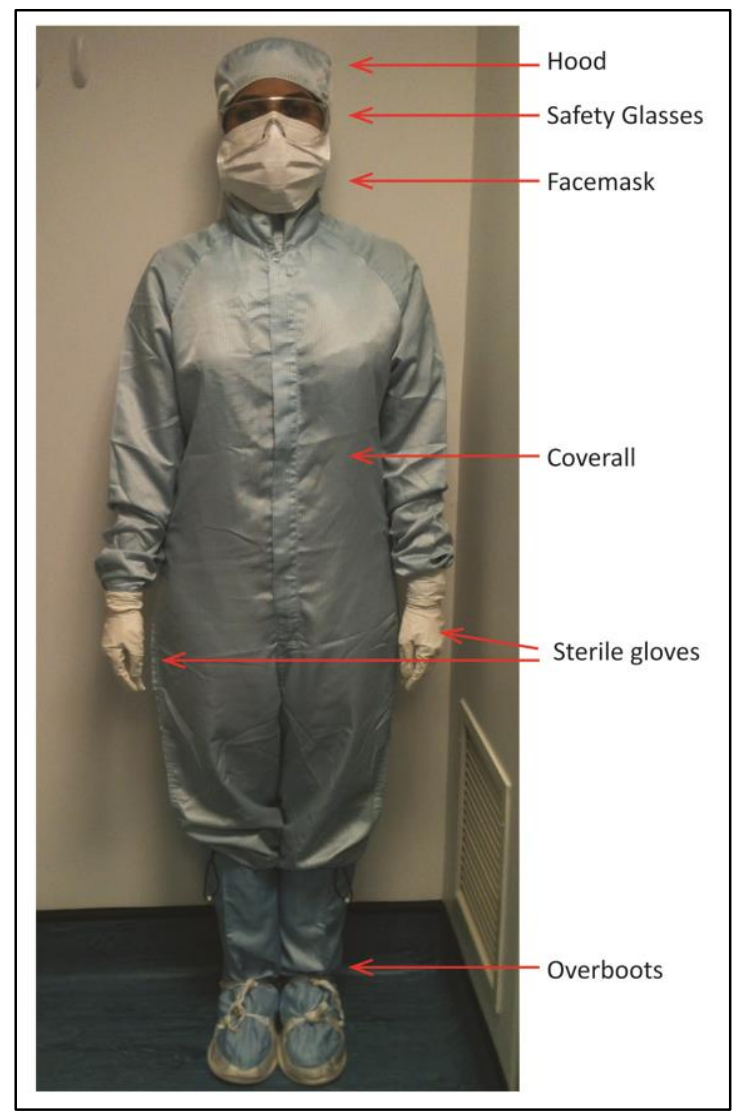

Figure 3: Garments required to operate / process at or above grade B. Operators are required to remove outdoor shoes and clothes and change into cleanroom tunics, trousers and clogs, and the donning of gloves and a mobcap (not shown above) prior to entering the facility. A second level of gowning is then performed prior to the operator moving to the Grade B laboratories, this involves putting on fresh gloves, a hood, safety glasses, a face mask, coverall and overboots, all of these items will have been sterilised by irradiation. 


\section{Quality Management System Overview}

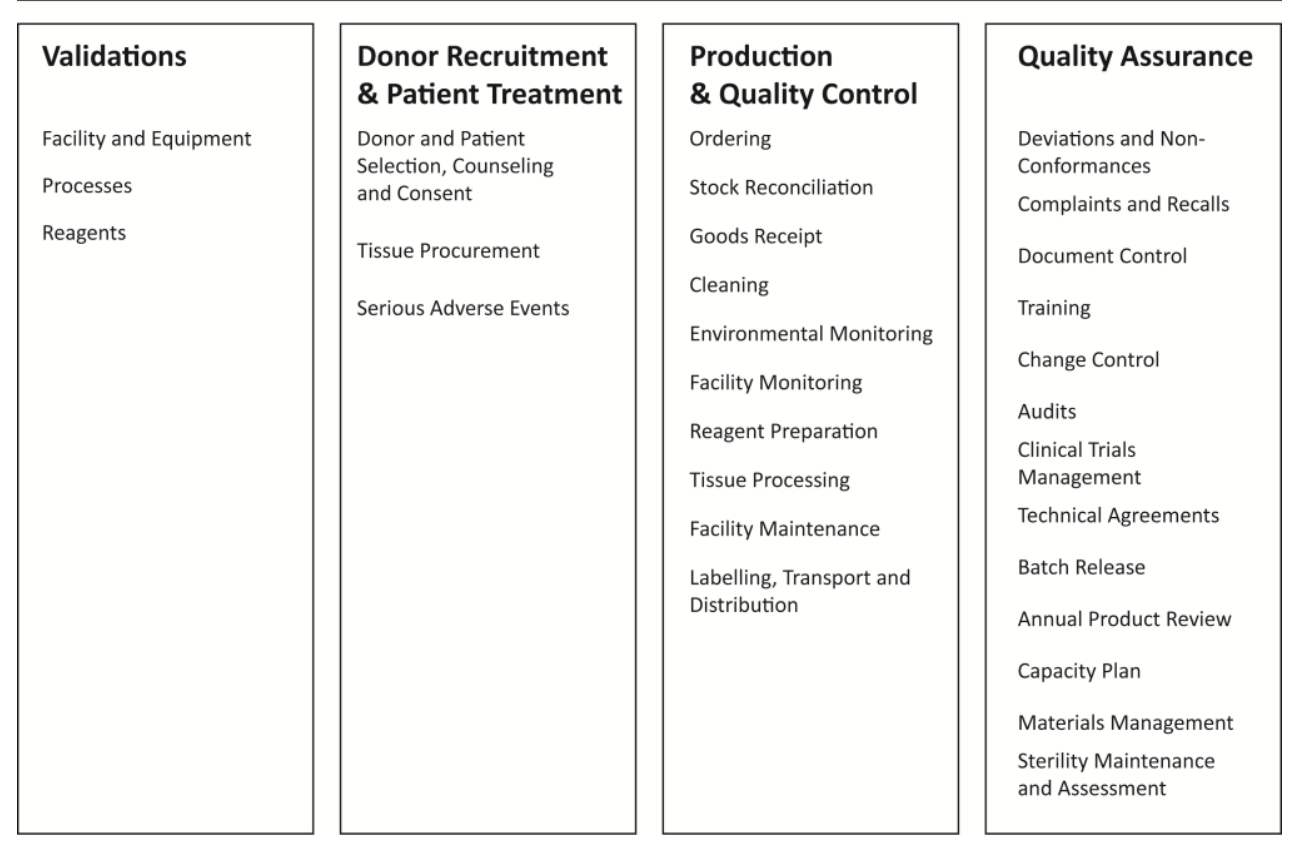

Figure 4: The CFS Quality Management System. The CFS QMS is divided into Validations, donor recruitment and patient treatments, production and quality control and quality assurance. 


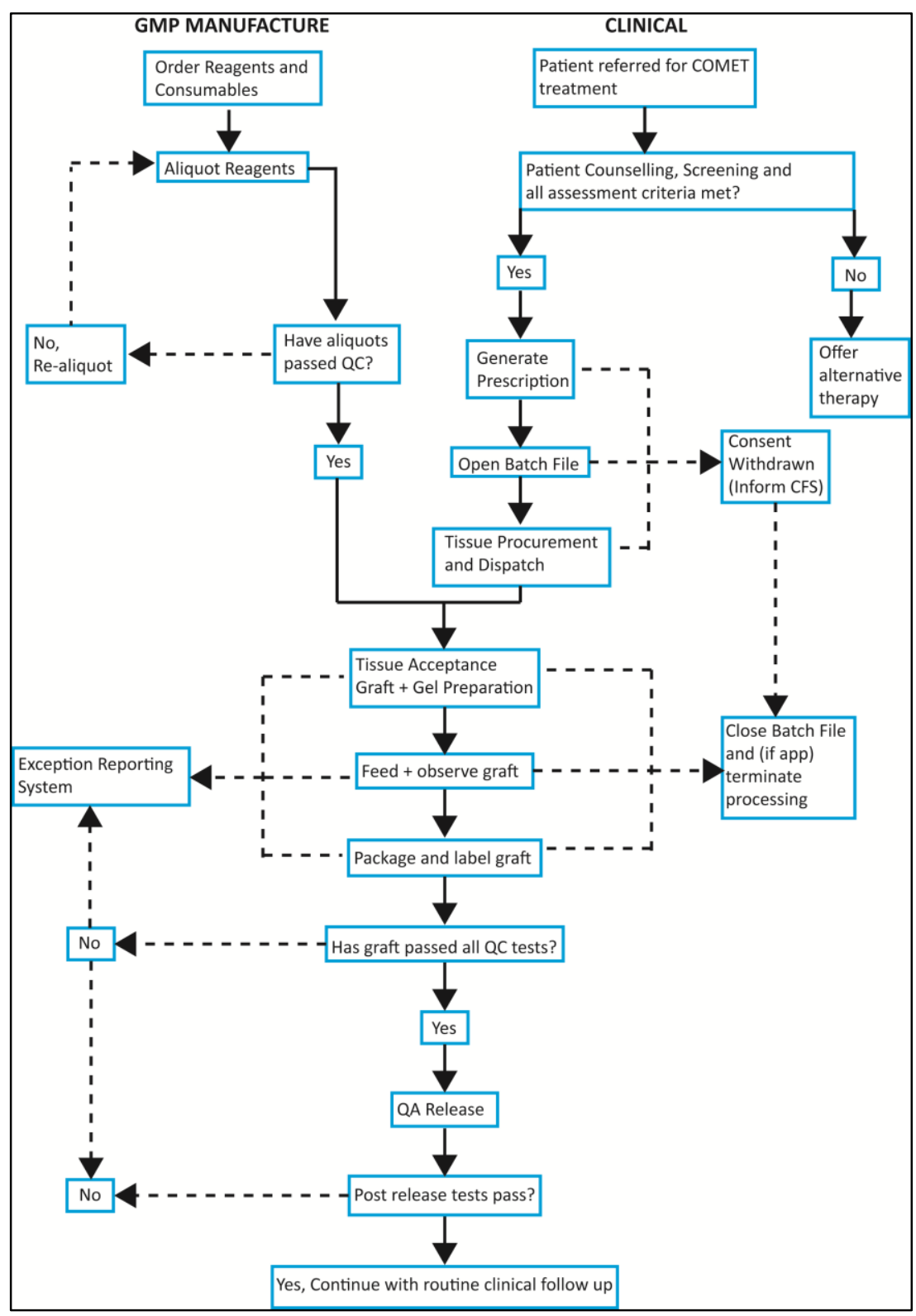

Figure 5: Process flow for the production of cultured epithelial cell grafts at CFS. The process flow diagram illustrates the steps involved in the production of cultured epithelial cell grafts at CFS. It encompasses all the quality, production and clinical requirements essential in the production of a GMP compliant cell therapy graft. 


\section{TABLE LEGENDS}

Table1: Cleanroom classification based on the maximum number of permitted particles/ $\mathbf{m}^{3}$. The classification of cleanrooms includes maximum particles per metre cubed of the 0.5 micron and the 5.0 micron particles. Usually there are two sets of levels allowed, one is for in-process and one is for at-rest [24].

Table2: Practical application of the different air grades of the cleanroom. The different air grades within the cleanroom are suitable for performing different processes. Each processing operation should be carefully planned based on the grade it will be performed in [24].

Table3: Recommended limits for microbial contamination. The classification of cleanrooms includes maximum CFU (Colony forming unit). Usually there are two sets of levels allowed, one is for in-process and one is for at-rest [24].

\section{ABBREVIATIONS:}

\begin{tabular}{|l|l|}
\hline GMP & Good Manufacturing Practice \\
\hline CFS & Cells For Sight \\
\hline UCL & University College London \\
\hline RM & Regenerative Medicine \\
\hline EUTCD & European Union Tissue and Cells Directive \\
\hline MHRA & $\begin{array}{l}\text { Medicines Healthcare and Regulatory products } \\
\text { Agency }\end{array}$ \\
\hline ATMP & Advanced Therapy Medicinal Products \\
\hline HTA & Human Tissue Authority \\
\hline VMD & Veterinary Medicines Directive \\
\hline MA & Marketing Authorization \\
\hline IMP & Investigative Medicinal Products \\
\hline QC & Quality Control \\
\hline QP & Qualified Person \\
\hline IMPD & Investigative Medicinal Products Dossier \\
\hline HVAC & Heating, Ventilation and Air Conditioning \\
\hline HEPA & High Efficiency Particulate Air \\
\hline AHU & Air handling Unit \\
\hline QA & Quality Assurance \\
\hline QMS & Quality Management System \\
\hline IPA & IsoPropyl Alcohol \\
\hline TSA & Tryptone Soya Agar \\
\hline SDA & Sabaroud Dextrose Agar \\
\hline EM & Environmental Monitoring \\
\hline CFU & Colony Forming Unit \\
\hline TSB & Tryptone Soya Broth \\
\hline SDB & Sabaroud Dextrose Broth \\
\hline ISO & International Organisation for Standardisation \\
\hline ICH & International Conference on Harmonisation \\
\hline & \\
\hline
\end{tabular}




\begin{tabular}{|l|l|}
\hline PPM & Planned Preventative Maintenance \\
\hline CAPA & Corrective And Preventative Actions \\
\hline SOP & Standard Operating Procedure \\
\hline SLA & Service Level Agreement \\
\hline TSE & Transmissible Spongiform Encephalopathy \\
\hline LSCD & Limbal Stem Cell Deficieny \\
\hline LEC & Limbal Epithelial Cells \\
\hline HIV & Human Immunodeficiency Virus \\
\hline
\end{tabular}

